\section{Digitale Versorgung-Gesetz: Vorstellungen des Gesetzgebers zur Digitalisierung des Gesundheitswesens}

Neben der öffentlichkeitswirksamen und hitzigen Diskussion um die Einführung einer Impflicht, die Widerspruchslösung im Rahmen der Organspende und dem Terminservice- und Versorgungsgesetz (TSVG) blieb weitgehend unbemerkt, dass das Bundesministerium für Gesundheit in Gestalt eines Referentenentwurfs für ein Digitale Versorgung-Gesetz initiativ in einem weiteren Bereich des Gesundheitswesens tätig geworden ist. Dieser Entwurf baut die mit dem Gesetz für sichere digitale Kommunikation und Anwendungen im Gesundheitsbereich (sog. E-Health-Gesetz) vom 28.12.2015 geschaffenen Instrumente (Videosprechstunde, elektronische Gesundheitskarte mit elektronischer Patientenakte) weiter aus und ergänzt diese um weitere Instrumente.

Den Referentenentwurf des BMG hat das Bundeskabinett am 10.7.2019 verabschiedet, der Bundestag hat sich hiermit noch nicht befasst; dennoch lohnt sich ein genauerer Blick in den Entwurf dieses Gesetzes für eine bessere Versorgung durch Digitalisierung und Innovation.

Das Gesetz sieht eine Reihe von Maßnahmen vor, die den Zugang zu digitalen Innovationen in der Regelversorgung erleichtern und die Entwicklung innovativer telemedizinischer Versorgungsangebote forcieren sollen. Zudem sollen die Telematikinfrastruktur ausgebaut und gleichzeitig neue Impulse für die Entwicklung innovativer Versorgungsansätze gegeben werden.

\section{Digitale Gesundheitsanwen- dungen}

Versicherte sollen künftig Anspruch auf Versorgung mit Medizinprodukten niedriger Risikoklasse haben, deren Hauptfunktion wesentlich auf digitalen Technologien beruht und die dazu bestimmt sind, bei den Versicherten oder in der Versorgung durch Leistungserbringer die Erkennung, Überwachung, Behandlung oder Linderung von Krankheiten oder die Erkennung, Behandlung, Linderung oder Kompensation von Verletzungen oder Behinderungen zu unterstützen (sogenannte digitale Gesundheitsanwendungen). Die Hersteller solcher digitalen Gesundheitsanwendungen sollen diese den Versicherten im Wege elektronischer Übertragung über öffentlich zugängliche Netze oder auf maschinell lesbaren Datenträgern zur Verfügung stellen. Verordnungsfähig sind dabei nur diejenigen Anwendungen, die in das Verzeichnis für digitale Gesundheitsanwendungen aufgenommen worden sind. Dieses Verzeichnis wird beim Bundesinstitut für Arzneimittel und Medizinprodukte (BfArM) geführt. Die in das Verzeichnis aufgenommenen digitalen Gesundheitsanwendungen sind im Bundesanzeiger bekannt zu machen und im Internet zu veröffentlichen. Aufgenommen wird eine digitale Gesundheitsanwendung in dieses Verzeichnis auf elektronischen Antrag des Herstellers beim Bundesinstitut für Arzneimittel und Medizinprodukte, sofern die Erfüllung der Grundanforderungen an Sicherheit, Funktionstauglichkeit und Qualität der Anwendung sowie deren positive Versorgungseffekte nachgewiesen sind. Das BfArM entscheidet über den Antrag des Herstellers innerhalb von drei Monaten nach Eingang der vollständigen Antragsunterlagen durch Bescheid. Ist der Nachweis positiver Versorgungseffekte durch die digitale Gesundheitsanwendung bei Antragstellung noch nicht möglich, kann die vorläufige Aufnahme in das Verzeichnis der digitalen Gesundheitsanwendungen für bis zu zwölf Monate zur Erprobung erfolgen. Einzelheiten werden durch eine Rechtsverordnung geregelt.

Die Krankenkassen werden ermächtigt, Verträge mit Herstellern über die besondere Versorgung der Versicherten mit digitalen Gesundheitsanwendungen abzuschlieBen. Hierbei ist die Einbindung ärztlichen Sachverstands sicherzustellen.

Damit werden sogenannte GesundheitsApps, die insbesondere bei der Behandlung von chronischen Krankheiten eine Rolle spielen können, in der gesetzlichen Krankenversicherung verordnungs- und erstattungsfähig.

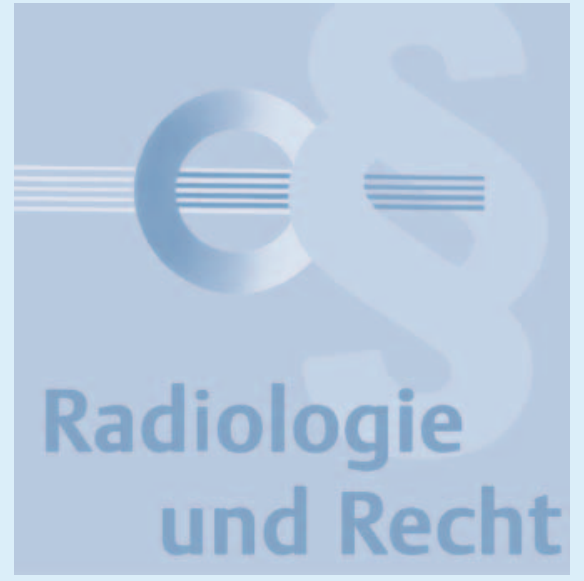

Um zu gewährleisten, dass die digitalen Gesundheitsanwendungen zweckentsprechend zur Unterstützung der vertragsärztlichen oder sonstigen Gesundheitsversorgung angewendet werden, soll die Erstattungsfähigkeit grundsätzlich zwar die ärztliche Verordnung voraussetzen. Allerdings soll die Erstattungsfähigkeit auch in den Fällen gegeben sein, in denen Versicherte digitale Gesundheitsanwendungen auf eigene Initiative oder Empfehlung anderer Leistungserbringer anwenden, also auch dann, wenn keine Verordnung seitens des behandelnden Vertragsarztes vorliegt. Voraussetzung hierfür ist jedoch, dass die jeweilige Krankenkasse für ihre Mitglieder die Zweckmäßigkeit der Anwendung bestätigt. Damit wird für die digitalen Gesundheitsanwendungen der Grundsatz durchbrochen, dass die Erstattung der Kosten eines Medizinprodukts, Heil- oder Hilfsmittels stets an eine ärztliche Verordnung geknüpft ist. Begründet wird dies damit, die Eigenständigkeit des Versicherten solle gestärkt werden, dieser solle seine Versorgung auch im Rahmen der ärztlichen Behandlung durch einen eigenverantwortlichen Beitrag aktiv mitgestalten können.

Für Radiologen werden digitale Gesundheitsanwendungen in der täglichen Patientenversorgung wohl eine eher untergeordnete Rolle spielen. Derartige Gesundheitsanwendungen werden im Regelfall nicht durch den diagnostisch tätigen Radiologen verordnet, sondern durch überweisende Ärzte. Allerdings könnten digitale Gesundheitsanwendungen z. B. für Strahlentherapeuten im Rahmen der onkologischen Patientenversorgung erhebliche Bedeutung erlangen - hier bleiben konkrete Entwicklungen abzuwarten. 


\section{Erweiterung der Telematikinfrastruktur}

Ein weiterer Schwerpunkt des Gesetzentwurfs ist die Erweiterung der Telematikinfrastruktur. Apotheken müssen sich bis zum 31.03.2020 und Krankenhäuser bis zum 01.03.2021 an die Telematikinfrastruktur anbinden. Für Pflege- und Rehabilitationseinrichtungen setzt der Gesetzgeber auf das Prinzip der freiwilligen Anbindung und bietet als Anreiz eine Erstattung der Ausstattungs- und Betriebskosten.

\section{Elektronische Patientenakte}

Darüber hinaus sieht der Referentenentwurf den Anspruch der Versicherten vor, dass ihre medizinischen Daten aus der vertragsärztlichen Versorgung ab dem 01.01.2021 in einer elektronischen Patientenakte gespeichert werden. Die elektronische Patientenakte ist eine versichertengeführte elektronische Akte. Sie wird als Anwendung der elektronischen Gesundheitskarte von der Gesellschaft für Telematik zugelassen und von den Krankenkassen ihren Versicherten zur Verfügung gestellt. Mit ihr sollen den Versicherten auf Wunsch medizinische Informationen, insbesondere zu Befunden, Diagnosen, durchgeführten und geplanten Therapiemaßnahmen sowie Behandlungsberichte für eine Einrichtungs-, Fach- und sektorenübergreifenden Nutzung elektronisch bereitgestellt werden. Versicherte sollen auch selbst Daten in ihrer elektronische Patientenakte hinterlegen können. Bis zum 31.03.2021 soll die Gesellschaft für Telematik die technischen Voraussetzungen dafür schaffen, die Kassenärztliche Bundesvereinigung soll im Benehmen insbesondere mit den Spitzenorganisationen der Krankenkassen und der Gesellschaft für Telematik die notwendigen Festlegungen für die Inhalte der elektronische Patientenakte treffen. Die Information der Versicherten über die Funktionsweise der elektronischen Patientenakte obliegt den Krankenkassen. Die an der vertragsärztlichen Versorgung teilnehmenden Leistungserbringer haben die Versicherten im Rahmen der vertragsärztlichen Versorgung über den Anspruch auf Speicherung der Daten in der elektronischen Patientenakte zu informieren und die Daten auf Wunsch des Versicherten in die elektronische Patientenakte einzustellen. Zudem haben sie die
Versicherten auf ihren Wunsch bei Anlage und Verwaltung der elektronischen Patientenakte zu unterstützen. Diese Aufgabe kann auf Personen, die als berufsmäßige Gehilfen oder zur Vorbereitung auf den Beruf bei dem jeweiligen Leistungserbringer tätig sind, übertragen werden. Die an der vertragsärztlichen Versorgung teilnehmenden Leistungserbringer haben gegenüber der jeweils zuständigen Kassenärztlichen Vereinigung nachzuweisen, dass sie über die für den Zugriff auf die elektronische Patientenakte erforderlichen Komponenten und Dienste verfügen. Wird dieser Nachweis nicht bis zum 30.06.2021 erbracht, soll die Vergütung vertragsärztlicher Leistungen pauschal um ein Prozent solange gekürzt werden, bis der Nachweis gegenüber der Kassenärztlichen Vereinigung erbracht wird.

Angesichts der Komplexität derartiger Telematikstrukturen sieht der Gesetzentwurf sinnvollerweise vor, dass das Bundesministerium für Gesundheit diese Frist durch Rechtsverordnung mit Zustimmung des Bundesrates verlängern kann.

Ebenfalls ab dem 01.01.2021 haben Versicherte Anspruch auf Speicherung ihrer im Rahmen einer Krankenhausbehandlung entstandenen Daten in der elektronischen Patientenakte.

Die Regelungen bezüglich der elektronischen Patientenakte betreffen auch das Fachgebiet der Radiologie: Der Gesetzentwurf unterscheidet nicht zwischen dem unmittelbar mit der Versorgung des Patienten befassten Arzt und dem auf Überweisung tätig werdenden Leistungserbringer. Auch Radiologen können also Adressaten des Wunsches von Patienten sein, bestimmte Daten oder Bilder in elektronische Patientenakten einzustellen. Ob Versicherte sich an Radiologen wenden werden, um sich von diesen bei Anlage und Verwaltung elektronischer Patientenakten unterstützen zu lassen, ist wohl nicht zu erwarten; hier dürfte faktisch eher der überweisende Hausarzt Ansprechpartner sein. Die elektronische Patientenakte ersetzt gleichwohl die bisherige Dokumentation der Behandlung durch den Radiologen nicht. Die elektronische Patientenakte ist lediglich eine an die elektronische Gesundheitskarte gebundene Dokumentation, die - da der Inhalt vom Wunsch des Patienten auf Speiche- rung von Daten abhängig und vor nachträglichen Änderungen nicht geschützt ist - nicht die Gewähr der Vollständigkeit bietet. Die Behandlung eines Patienten allein auf die Daten der elektronischen Patientenakte aufzubauen, kann daher unter Umständen ein Haftungsrisiko für den behandelnden Arzt begründen. Ebenso kann sich ein Haftungsrisiko für den Arzt ergeben, dem bei Speicherung von Daten in der elektronischen Patientenakte ein Fehler unterläuft.

\section{Videosprechstunde}

Ferner sieht das Gesetz eine Stärkung telemedizinischer Verfahren zur effizienten und schnelleren fachlichen Zusammenarbeit von Vertragsärzten und mit anderen Ärztinnen und Ärzten, etwa in Ambulanzen und den Krankenhäusern, vor. Zu diesem Zweck sollen z. B. Tele-Konsile in einem weiten Umfang in der vertragsärztlichen Versorgung und sektorenübergreifend ermöglicht und extrabudgetär vergütet werden. Hierzu hat der Bewertungsausschuss innerhalb von neun Monaten nach Inkrafttreten des Gesetzes entsprechende Anpassungen zu beschließen. Die bereits bestehenden Möglichkeiten der Inanspruchnahme einer Videosprechstunde werden durch weitere gesetzliche Regelungen vereinfacht. So wird klargestellt, dass eine in einem Einzelfall erforderliche Aufklärung und Einwilligung im Rahmen telemedizinischer Behandlung auch unter Einsatz der für die telemedizinische Behandlung verwendeten Fernkommunikationsmittel erfolgen kann.

\section{Digitalisierung von Verwal- tungsabläufen; Förderung digitaler Innovationen}

Um den Einsatz elektronischer Arztbriefe zu fördern, wird die Vergütung des Telefax im einheitlichen Bewertungsmaßstab reduziert. Die Organe der Selbstverwaltung werden zudem verpflichtet, die erforderlichen Regelungen zur Verwendung von Verordnungen in elektronischer Form für HeilHilfsmittel zu schaffen. Hierdurch sollen Verwaltungsprozesse durch Digitalisierung vereinfacht werden. Schließlich erhalten die Krankenkassen erweiterte Möglichkeiten, die Entwicklung digitaler Innovationen 
im Gesundheitswesen (darunter der Einsatz digitaler Medizinprodukte, künstlicher Intelligenz, telemedizinischer oder IT gestützter Verfahren) zu fördern. Zu diesem Zweck sollen die Innovationsfonds bei den Krankenkassen ausgebaut werden.

\section{Fazit}

Aus der Sicht des in der Patientenversorgung tätigen Arztes werden insbesondere die digitalen Gesundheitsanwendungen (Gesundheits-Apps) sowie die Regelungen zur Führung der elektronischen Patientenakte von Bedeutung sein. Allerdings lässt sich derzeit noch nicht absehen, wann das Gesetz in Kraft treten wird (hiervon sind die Bezugsdaten zur Schaffung der telemedizinischen Rahmenbedingungen abhängig) und ob die komplexen Abstimmungsprozesse zwischen den an dem Aufbau der Telematikstruktur Beteiligten tatsächlich innerhalb der vom Gesetzgeber im Gesetzentwurf vorgesehenen eher kurz gehaltenen Fristen so zu erledigen sind, dass ausgereifte Telematikstrukturen im Rahmen der vertragsärztlichen Versorgung tatsächlich zur Verfügung stehen. Dennoch zeigt der Gesetzentwurf, dass telemedizinische Strukturen zunehmend im Rahmen des Arzt-Patientenverhältnisses an Bedeutung gewinnen werden. Für den Arzt folgt daraus, dass er häufiger auf Patienten treffen wird, die den Einsatz derartiger Telematik- strukturen in der vertragsärztlichen Versorgung erwarten. Der Gesetzentwurf ist damit auch ein Hinweis an die Ärzteschaft, sich frühzeitig auf die verändernden Rahmenbedingungen in der vertragsärztlichen Versorgung einzustellen.

\section{Dr. Horst Bonvie \\ Rechtsanwalt}

Rechtsanwälte Wigge

Großer Burstah 42

20457 Hamburg

Telefon: (040) 3398 705-90

Telefax: (040) 3398 705-99

Internet: www.ra-wigge.de

E-Mail: kanzlei@ra-wigge.de 